Revue d'histoire de l'enfance « irrégulière »

Le Temps de l'histoire

1 | 1998

La protection de l'enfance : regards

\title{
Evolution actuelle de la Justice des mineurs : sa place dans la cité
}

Alain Bruel

\section{(2) OpenEdition \\ 12 Journals}

Édition électronique

URL : http://journals.openedition.org/rhei/19

DOI : $10.4000 /$ rhei. 19

ISBN : 978-2-7535-1638-0

ISSN : $1777-540 \mathrm{X}$

Éditeur

Presses universitaires de Rennes

Édition imprimée

Date de publication : 15 novembre 1998

Pagination : 109-117

ISSN : 1287-2431

Référence électronique

Alain Bruel, «Evolution actuelle de la Justice des mineurs : sa place dans la cité », Revue d'histoire de l'enfance « irrégulière » [En ligne], 1 | 1998, mis en ligne le 02 mai 2007, consulté le 03 décembre 2020 URL : http://journals.openedition.org/rhei/19 ; DOI : https://doi.org/10.4000/rhei.19 


\section{Évolution actuelle de la Justice des mineurs Sa place dans la cité}

Pour esquisser les grandes lignes du sujet, il m'a paru indispensable d'opérer une mise en perspective historique. Contraint de toute façon à simplifier, j'ai choisi arbitrairement de reprendre cinq idées-force de l'ordonnance de $1945,{ }^{(3)}$ d'étudier dans quelle mesure leur approche avait évolué en 1983, lorsque la commission Martaguet ${ }^{(4)}$ a déposé son rapport, et comment elles sont interprétées à l'heure actuelle.

Pourquoi 1945? En raison bien sûr de son aspect fondateur, mais aussi parce que, on le sait maintenant, l'ordonnance de $1958,{ }^{(5)}$ qui a marqué l'essor de l'assistance éducative, en constituait en quelque sorte le deuxième volet et était en tout cas issue du même moule.

Pourquoi la commission Martaguet? En raison de l'étendue de son champ de réflexion, de sa composition pluridisciplinaire et surtout de la qualité exceptionnelle de son animation par le Premier président Martaguet, formé à l'école de la dynamique de groupe et qui a su faire rendre à son équipe le maximum dont elle était capable compte tenu des idéologies qui avaient cours à l'époque.

Au demeurant, si ses idées ont, dans un premier temps, pâti du climat politique de l'époque et de la mise en place de la cohabitation, elles ont ensuite largement inspiré, et inspirent encore comme on le verra, la réflexion actuelle.

\section{1 - LA RESPONSABILITÉ DU MINEUR}

L'ordonnance de 1945 était profondément marquée par les idées de la Défense sociale nouvelle qui appréhendait essentiellement la délin-

\section{Alain Bruel $^{(2)}$}

(1) Ce texte est la communication de l'auteur aux Journées d'études L'Éducation surveillée aurait eu 50 ans, Vaucresson, les 9 et 10 novembre 1995.

(2) Président du tribunal pour enfants de Paris.

(3) Ordonnance du 2 février 1945 sur l'enfance délinquante.

(4) Commission de réforme du droit pénal des mineurs, présidée par M. Martaguet (1983).

(5) Ordonnance du 23 décembre 1958 sur l'enfance en danger. 
quance comme le "symptôme » de déficiences bio-psychologiques, sociales ou relationnelles. Elle a donc fait justice de la question du discernement qui avait montré ses insuffisances et posé le principe général de «l'irresponsabilité pénale » des mineurs.

Principe absolu ou relatif selon l'âge, mais fondamental dans la mesure où, face à la délinquance, la réponse normale ne devait plus être la peine, mais la mesure éducative. Fort logiquement, l'ordonnance de 1958, en s'attaquant à la notion de «danger » quelle qu'en soit la forme et l'origine, a cherché par la suite à améliorer l'efficacité du dispositif en s'attaquant, à travers des symptômes moins formels que ceux répertoriés dans le code pénal, aux situations qui pouvaient apparaître comme génératrices à terme de délinquance. On parlait alors de "prédélinquance ".

En 1983, la pensée parait avoir pris une direction radicalement inverse, puisque c'est au contraire le principe de la « responsabilité $d u$ mineur " qui est affirmé.

Entre temps, éducateurs et juges ont commencé à s'apercevoir que l'acte ne peut être réduit à un symptôme. Il n'est pas indifférent que l'individu ait adopté tel ou tel type de réaction. Il y a là, qu'on le veuille ou non, un certain choix qui lui est personnel et qu'on ne peut lui retirer sans le nier dans sa singularité. Certains psychologues, à commencer par Jacques Selosse, voient même dans le délit une interpellation adressée à la société par un adolescent en recherche identitaire.

À y regarder de près, les conclusions qu'en tire la commission Martaguet n'en sont pas orientées pour autant sur un recours accru à la répression.

Il s'agit plutôt d'un affinement des conceptions éducatives.

À l'heure actuelle, le souci de responsabilisation perdure. Mais quelque chose a profondément changé : on a pris conscience entre temps de l'impossibilité de considérer le jeune délinquant simplement comme un sujet au sens psychologique du terme. Les déboires enregistrés, un certain divorce avec l'opinion publique et surtout la massification de certains comportements délinquants évoquent moins la pathologie individuelle qu'une "crise du lien social " qu'on a le devoir de restaurer. Aussi le centre d'intérêt se déplace-t-il sur le "sujet de droit ». L'impact de la convention des Nations unies sur les droits de l'enfant y contribue, ainsi 
que la réflexion sur l'appartenance généalogique et culturelle. Responsabiliser, c'est instituer l'individu par rapport à ses droits et ses devoirs.

\section{2 - LE PRIVILÈGE DE JURIDICTION}

L'ordonnance de 1945 avait prévu pour le mineur un privilège de juridiction, c'est-à-dire la certitude d'avoir affaire, tout au long du processus pénal, non pas aux juridictions ordinaires, mais à un magistrat spécialisé. Spécialisation et continuité personnelle qui se sont incarnées dans le personnage emblématique du juge des enfants.

L'ordonnance de 1958 a poussé jusqu'au bout la logique du système, en faisant de ce même magistrat l'unique maître d'œuvre de la protection.

La durée des mesures qu'il ordonne est, à l'époque, indéterminée. Elles peuvent perdurer jusqu'à la majorité qui est de 18 ans au pénal, mais intervient seulement à 21 ans au civil.

En 1983 la commission Martaguet maintient le principe, mais, dans le dessein d'en renforcer l'efficacité, elle décide de l'étendre et avance la notion d'"équipe judiciaire ". Il en résulte une disparition du monopole de fait du juge des enfants; l'accent est mis sur la nécessaire complémentarité de ses interventions avec celles du parquet des mineurs, du juge d'instruction spécialisé et même du défenseur, dont la spécialisation est elle aussi souhaitée.

À l'heure actuelle ces idées se sont largement concrétisées au moins en ce qui concerne le parquet et la défense.

Mais, si personne ne discute le principe du polycentrisme et de la complémentarité des tâches, le problème est celui de leur répartition.

La montée en puissance du parquet dans un contexte répandu d'asphyxie de la juridiction, son intervention en amont de celle du siège, suscitent parfois des excès. D'où l'importance de la notion d'équilibre fonctionnel.

Le développement du partenariat inter-institutionnel dans le cadre de la politique de la ville parait à l'heure actuelle un domaine d'élection pour le parquet. 
Ses essais dans le domaine des placements d'urgence et des médiations pénales, parfois moins concluants, posent de difficiles questions de principe : le traitement individualisé dans la durée reste bien l'apanage du juge des enfants. À cet égard, les doutes émis dans un arrêt de la Cour de Reims sur l'impartialité d'un magistrat qui juge après avoir instruit paraissent avoir été provisoirement balayés par la Cour de cassation.

\section{3 - LA PROCÉDURE INQUISITOIRE}

Désireuse de faciliter au juge une connaissance approfondie de la personnalité des mineurs dont il avait la charge, l'ordonnance de 1945 avait eu le souci de briser tous les carcans du formalisme, en même temps qu'elle rendait obligatoires certaines investigations comme l'enquête sociale; les procédures expéditives du flagrant délit et de la citation directe étaient interdites. Parallèlement, le souci de la confidentialité des échanges transparaissait avec les règles de publicité restreinte et la possibilité d'épargner aux mineurs, en les faisant sortir à certains moments du débat, le traumatisme de révélations susceptibles de les troubler.

L'ordonnance de 1958 a porté à l'extrême ces principes inquisitoriaux et renchéri sur la souplesse au point de susciter l'indignation des juristes classiques et de provoquer la réaction de 1970 plus protectrice de l'autorité parentale.

En 1983, si la souplesse de la procédure continue à apparaître comme un acquis positif, l'organisation du contradictoire, la nécessité d'une défense se font jour; on voit aussi apparaitre au pénal la notion de "délai raisonnable » de jugement, grâce à un mécanisme d'impossibilité de sanctionner au-delà d'un an après la saisine.

Mais la confidentialité de la procédure n'est pas mise en cause.

De nos jours, les notions de "débat contradictoire », de "transparence", de "nécessaire continuité de la défense » ont fait leur chemin. Les juges s'efforcent, avec plus de scrupules que par le passé, d'enrichir la contradiction en multipliant les auditions des mineurs, pères et mères, mais aussi en poussant les inspecteurs de l'Aide sociale et les travailleurs sociaux, voire même les psychologues, à soutenir leur point de vue à l'audience. 
Devenue obligatoire au pénal, la présence des avocats est moins rare en assistance éducative. Il arrive même que la désignation d'un défenseur de l'enfant soit suscitée par le magistrat. Il se pourrait d'ailleurs que le mouvement s'accélère et que le principe de confidentialité lui-même soit mis en cause suite à la décision de la Cour européenne, en date du 24 février 1995, qui, à partir de la notion de "procès équitable ", a condamné l'Écosse au motif que "la non-communication des rapports sociaux affectait la capacité des parents d'influer sur l'issue de l'audience».

\section{4 - L'UNITÉ DES TRAITEMENTS CIVIL ET PÉNAL}

L'ordonnance de 1945 et celle de 1958 reposaient l'une et l'autre sur le postulat fondamental de l'unité de l'inadaptation; ce concept flou, mélangeant les catégories morales, légales, sociales et cliniques, présentait au moins l'avantage de ne pas isoler la délinquance par rapport aux autres manifestations de malaise de l'adolescent.

Cette conviction qu'il s'agissait bien, dans un cas et dans l'autre, de réactions différentes répondant à des difficultés comparables a permis la dépénalisation de certains comportements comme la fugue, la prostitution ou l'usage occasionnel de drogue, récupérés par l'assistance éducative au même titre que l'absentéisme scolaire ou les mauvais traitements.

En 1983, elle n'a nullement été remise en cause; cependant, depuis quelques années déjà, on critiquait l'ouverture jugée trop fréquente des doubles dossiers civil et pénal pour un même mineur. Ce phénomène imputé à la relative rigidité de l'ordonnance de 1945, notamment quant au prononcé de placements définitifs, était certes considéré comme une déviation, mais trop utile pour qu'on en préconise la suppression.

La réflexion moderne est plus critique : la pratique des doubles dossiers est périodiquement attaquée au nom d'impératifs gestionnaires, le financement étant, au pénal, à la charge exclusive de l'État et, au civil, principalement à la charge du département.

Par ailleurs, l'opinion publique, la presse, voire même quelques professionnels manifestent une propension certaine à distinguer mineurs en danger et mineurs dangereux. Le recentrage de l'activité des juges des 
enfants sur le pénal, qu'ils avaient quelque peu délaissé, ne doit pas, à mon sens, aller jusqu'à une telle dérive, grosse de dangers incalculables pour la protection des mineurs.

Reste que la mise à nu de la pratique des doubles dossiers, qui présente à la fois des avantages (intervention continue, rationnelle, affranchie de la temporalité artificielle des passages à l'acte; prise de recul permettant de choisir le moment de juger; moindre stigmatisation sociale du délinquant) et des inconvénients (éparpillement des données de personnalité, invisibilité du traitement éducatif dans l'activité pénale, manque de considération pour la victime), fait apparaître un besoin de réforme.

\section{5 - LA PRIMAUTÉ DE L’ACTION ÉDUCATIVE}

En affirmant la primauté de l'action éducative et en reléguant la peine dans une fonction résiduelle, l'ordonnance de 1945 postulait la notion d'éducabilité et une confiance inébranlable dans le caractère positif et l'efficacité des nouvelles mesures.

L'attitude de la commission Martaguet ne sera pas foncièrement différente. Cependant, avec trente ans de recul, l'enthousiasme des origines a fait place à une attitude plus défensive. Elle se manifeste par l'affirmation de l'autonomie de l'action éducative, qui doit être préservée de toute annexion à des fins répressives. On affirme que n'importe quelle éducation comporte une part de contrainte qui lui est intrinsèque.

En revanche, assortir la mesure éducative d'une menace à caractère pénal ou en faire l'instrument d'une surveillance pure et simple constituent autant de perversions. On refuse de sanctionner l'échec éducatif en tant que tel. On débat sur l'opportunité de créer entre l'action éducative et la peine un registre intermédiaire plus ou moins disciplinaire et on hésite sur le point de savoir à quels professionnels il pourrait être confié.

Enfin on voit apparaître fugitivement le concept d'incitation à la réparation. Celui-ci étant appelé à faire pendant à la recherche de l'adhésion en assistance éducative.

Le débat actuel s'est légèrement déplacé. Chacun s'accorde à reconnaître que l'action éducative "à l'ancienne", orientée sur la rééducation 
d'un individu pathologique ou déviant, ne répond plus aux attentes sociales ni aux besoins des mineurs. On s'oriente vers un modèle pragmatique d'accompagnement à l'insertion qui prend en compte la crise du lien social et s'articule à la politique de la ville.

On intègre mieux la décentralisation et la territorialisation de l'action éducative, mais celle-ci ne doit pas pour autant perdre de vue son objectif de construction de la personne. Pour échapper au danger d'arbitraire, elle doit aussi s'inscrire dans un double cadre : le judiciaire qui la légitime, lui fixe des limites dans le temps et quant au respect des libertés; le technique qui résulte d'une utilisation éclectique des sciences humaines.

Longtemps obscurci par une confusion avec la notion voisine de médiation, le débat sur la réparation pénale est enfin engagé.

Le caractère éducatif de la mesure a d'abord été contesté. Il est vrai que, réduite à une tâche de médiation confiée à un éducateur, rarement précédée par une audition au parquet, elle pouvait paraître peu conforme au principe d'autonomie de l'action éducative dégagé en 1983.

Analysée comme une injonction adressée au mineur et à sa famille, à propos de laquelle l'éducateur intervient surtout pour aider les intéressés à définir une offre et à en mener à bien la réalisation, ordonnée de plus en plus fréquemment après la mise en examen par un magistrat du siège et en présence de la défense, la réparation directe ou indirecte apparait beaucoup moins suspecte. Elle s'accorde parfaitement à l'approche du délinquant en tant que sujet de droit. Elle s'avère potentiellement très reconstructrice du lien social : d'objet passif de la sollicitude éducative, le mineur se trouve en effet promu en qualité d'acteur de sa réhabilitation; ses parents, autrefois pris en leur seule qualité de civilement responsables, redeviennent prioritairement les guides naturels de leurs enfants ; la victime est prise en considération non seulement comme titulaire d'un préjudice, mais comme être humain lésé physiquement et moralement.

Enfin la communauté sociale elle-même se trouve mobilisée, au moins dans la réparation indirecte, pour fournir le cadre d'accueil et d'encadrement nécessaire à la mise en œuvre de la mesure.

La seule précaution à prendre consiste à garder en mémoire que la réparation ne saurait épuiser toutes les dimensions de l'action éducati- 
ve au pénal, dont elle n'est qu'un élément privilégié, et qu'elle doit toujours, pour acquérir un sens, être assortie de larges possibilités de verbalisation.

\section{CONCLUSION}

Il convient, pour conclure, de dire quelques mots de la place actuelle de la justice des mineurs dans la cité et des réformes vers lesquelles nous devons nous acheminer.

Grâce aux analyses de certains philosophes du droit, sociologues et juristes, particulièrement Blandine Barret-Kriegel, on appréhende mieux aujourd'hui l'originalité du mode de décision judiciaire comparé au mode administratif. À la différence de ce dernier qui s'élabore de manière technocratique, confidentielle, autoritaire et pose des règles générales, la décision judiciaire procède d'un débat qui tend à devenir public; elle doit être motivée et viser le plus possible l'obtention d'un consensus, même si celui-ci ne lui est pas indispensable; elle ne peut en revanche appréhender que des situations individuelles.

Dès lors, on évalue la performance du juge à la qualité du débat qu'il organise. La juridiction des mineurs n'échappe pas à la règle.

Compte tenu de ses origines paternalistes et de l'évolution des exigences sociales, elle doit tendre à une meilleure visibilité de ses réponses.

Ce caractère, qui semble avoir pris la place de l'exemplarité, comporte deux exigences de rapidité et de clarté.

$\mathrm{Si}$, à première vue, ces qualités sont incompatibles avec le temps de maturation et d'élaboration nécessaire à l'action éducative, l'idée d'une « césure du procès pénal » évoquée par la commission Martaguet dès 1983 pourrait, par une articulation souple, permettre de résoudre cette apparente contradiction.

Il s'agirait d'obliger le juge à statuer dans des délais rapides sur la culpabilité de l'auteur, le préjudice subi par la victime et la responsabilité civile des parents, en lui laissant la faculté d'ajourner à 6 mois la décision sur la sanction, tout en ordonnant les investigations, actions éducatives et réparations qui pourraient s'avérer utiles. 
L'exigence de clarté est plus difficile à satisfaire, la visibilité sociale et le respect de l'intimité du sujet étant parfois incompatibles en l'état actuel des rapports psychologiques et sociaux annexés aux procédures civiles et pénales. On devrait donc tendre à distinguer les notions d'espace privé et d'espace public dont les frontières ont été bouleversées depuis le début du siècle par l'intervention sociale dans les familles, ainsi que l'a montré Irène Théry.

Mais peut-on si facilement dissocier la confidentialité de la relation clinique et le devoir de rendre compte de ce qui a trait au passage à l'acte et à l'état de danger?

Le deuxième changement qui paraît être exigé de la juridiction des mineurs porte sur ses liens avec l'extérieur.

Il est hors de doute qu’à la logique verticale de 1945 a succédé une logique horizontale de partenariat. Celle-ci met en lumière des défaillances de la prévention qui produisent, au civil, un encombrement du judiciaire et, à l'inverse, au pénal, des difficultés de transmission certaines, doublées d'une mauvaise connaissance par les juges du contexte social d'environnement dans lequel ils rendent leurs décisions.

On a déjà évoqué le rôle capital d'interface que commence à jouer le parquet des mineurs. Quant au rapprochement géographique des juges, fréquemment pressés d'investir les maisons de justice, on peut se demander s'il constituerait un progrès : une certaine prise de distance, un refus de banalisation sont indispensables à la tenue du rôle judiciaire au pénal. Pour remédier au manque d'information des magistrats, qui les conduit parfois à prendre des décisions d'inspiration psychologisante inadaptées à la réalité sociologique du terrain et de ce fait mal perçues, un double effort devrait être accompli par le ministère de l'Intérieur pour spécialiser certains policiers dans la recherche et le contact avec les jeunes délinquants et par le ministère de la Justice pour territorialiser l'action éducative.

Au niveau du SEAT, ${ }^{(6)}$ chaque secteur judiciaire devrait être couvert par un éducateur capable de représenter le judiciaire dans les instances locales de Prévention de la délinquance et de pouvoir rendre compte au juge de ce qui s'y fait et de ce qui s'y dit. 